\title{
The clinical utility of bronchoalveolar lavage in diffuse parenchymal lung disease
}

\author{
A.U. Wells
}

\begin{abstract}
Bronchoalveolar lavage (BAL) has been used in diagnostic and prognostic evaluation in diffuse parenchymal lung disease for three decades and has a central role in the diagnosis of a number of rare disorders and in excluding opportunistic infection in treated patients. It also has an important place in the personal diagnostic algorithms of many experienced clinicians in the diagnosis of the more prevalent disorders, including sarcoidosis, hypersensitivity pneumonitis and idiopathic pulmonary fibrosis. This use of BAL is not well captured in the medical literature, as most published studies pre-date changes in disease classification and fail to integrate BAL data with other clinical and radiological information. Further studies to quantify the value added by bronchoalveolar lavage in routine practice are urgently required.
\end{abstract}

KEYWORDS: Bronchoalveolar lavage, diffuse parenchymal lung disease, interstitial lung disease

T he diagnostic and prognostic utility of bronchoalveolar lavage (BAL) was first evaluated in historical series in the 1980s, and endorsed by expert groups [1, 2]. However, much has changed in the last two decades. In the last century, preceding the American Thoracic Society (ATS)/European Respiratory Society (ERS) reclassification of the idiopathic interstitial pneumonias (IIPs) [3, 4], diverse disease processes were amalgamated under the labels of "idiopathic pulmonary fibrosis" (IPF) or "cryptogenic fibrosing alveolitis". Amongst these disorders, cryptogenic organising pneumonia (COP) was described in the mid-1980s [5] but typical BAL profiles were not at first recognised and, thus, the presence of COP was not considered in the differential diagnosis of a lymphocytic profile. This omission had important implications with regard to reports of the diagnostic and prognostic utility of BAL.

Nonetheless, the early series drew important broad diagnostic distinctions between granulomatous lung disease (sarcoidosis, hypersensitivity pneumonitis (HP) and the fibrosing diseases broadly amalgamated historically as "IPF"). In fibrosing disorders, a BAL neutrophilia, often associated with an eosinophilia, is common. Granulomatous disease, by contrast, is characterised by the predominance of a BAL lymphocytosis, with a variable BAL neutrophil content and, in HP, an occasional BAL eosinophilia. This essential distinction has stood the test of time.
However, the use of BAL to make more refined diagnostic subdivisions amongst the more prevalent diseases has proved disappointing.

\section{TYPICAL BAL PROFILES IN SELECTED INDIVIDUAL DISEASES}

In IPF, the typical BAL profile is a neutrophilia, with or without an eosinophilia. A minority of patients have a mild BAL lymphocytosis, which seldom exceeds $25 \%$ of the differential cell count. This profile, first documented in historical "IPF", applies equally to IPF diagnosed using modern histological, radiological and clinical criteria. However, with ATS/ERS reclassification, and the use of high-resolution computed tomography (HRCT) to identify overtly fibrotic disease, it has become apparent that a BAL granulocytosis is highly nonspecific. For example, a BAL neutrophilia is evident in a subset of patients with sarcoidosis and there is indirect evidence that this denotes the presence of more extensive pulmonary fibrosis $[6,7]$. In chronic $\mathrm{HP}$, both granulocytic elements are variably present, with a BAL neutrophilia sometimes prominent [8].

The nonspecific nature of a BAL neutrophilia is illustrated by the difficult diagnostic problem of fibrotic nonspecific interstitial pneumonia (NSIP). Nearly 10 yrs ago, the clinical entity of NSIP was tional multidisciplinary consensus classification committee [4]. It subsequently became apparent that the term NSIP, as applied over the past termed as "provisional" by the ATS/ERS interna-
CORRESPONDENCE

A.U. Wells

Emmanuel Kaye Building

Manresa Rd

Chelsea

London

SW3 6LR

UK

E-mail: athol.wells@rbht.nhs.uk

Received:

May 252010

Accepted:

May 262010

PROVENANCE

Publication of this peer-reviewed article was supported by GlaxoSmithKline, Belgium (principal sponsor, European Respiratory Review issue 117). 
decade, covered distinct diverse clinico-radiological profiles, including profiles suggestive of COP, HP and even IPF [9]. Based on HRCT findings, it is clear that in early East Asian series, NSIP patients with variably extensive consolidation were studied and a prominent BAL lymphocytosis was the rule in both fibrotic and cellular NSIP $[10,11]$. By contrast, in a series of fibrotic NSIP patients without consolidation on HRCT, BAL profiles were, on average, identical to those in IPF, with lymphocytosis seldom present and neutrophilia the more prevalent abnormality [12].

Among the remaining IIPs, typical BAL profiles have been documented. In respiratory bronchiolitis-associated interstitial lung disease (RBILD), compared to other IIPs, there are significant increases in macrophage numbers and significantly lower percentages of other cellular components, with a BAL neutrophilia or eosinophilia virtually never present [12]. A characteristic brown pigmentation of alveolar macrophages is consistently observed in current smokers and this applies equally to RBILD, and to the subset of patients with desquamative interstitial pneumonia (DIP) ascribable to smoking. However, in DIP, cellular components other than macrophages are more likely to be increased, with BAL neutrophilia, eosinophilia and lymphocytosis variably present [12]. The BAL profile of COP, evaluated in a number of studies [13], includes a lymphocytosis in the great majority of cases, but other cell types (neutrophils, eosinophils and mast cells) are commonly (although not always strikingly) increased.

The use of BAL data to distinguish between sarcoidosis and HP remains contentious. In both diseases, increased numbers of T-lymphocytes are found in active disease. In both diseases, a granulocytosis is present in some cases. Sarcoidosis and HP cannot be infallibly distinguished by the quantity of any single cell type in BAL fluid. However, diagnostic probabilities are usefully refined by the consideration of grouped BAL features. An elevated total cell count consisting predominantly of lymphocytes is suggestive of sarcoidosis when the percentage of neutrophils and eosinophils is normal or near normal and plasma cells are absent [14, 15]. In contrast, although lymphocytes predominate in HP, the numbers of all cellular constituents tend to be increased substantially in active disease [8], with variable effects on neutrophil and eosinophil differential percentages. To complicate matters further, the BAL profile of HP is also heavily influenced by the time that has elapsed from antigen inhalation and the intensity of exposure.

The T-lymphocyte component in sarcoidosis and HP can be sub-categorised as predominantly T-helper (CD4) or T-suppressor (CD8) cells. In some centres, CD4/CD8 ratios are regarded as diagnostically useful, with an increased CD4/CD8 ratio favouring a diagnosis of sarcoid, whereas a low CD4/ CD8 ratio is more usual in HP $[16,17]$. It is clear that there are too many exceptions to these observations [18] to allow this BAL distinction to be definitive in isolation. However, nondefinitive tests may sometimes be invaluable, by virtue of shifting the balance of diagnostic probability in the right clinical setting. On this basis, CD4/CD8 ratios continue to have an important role in the personal diagnostic algorithms of many clinicians, although the best use of this test requires considerable experience in its application.
As detailed later, BAL findings are pathognomonic in some rare disorders. However, in the more common diseases (sarcodiosis, HP and the IIPs), BAL findings do not tend to be definitive in isolation, but need to be integrated with other data in order to be diagnostically useful.

\section{THE DIAGNOSTIC INTEGRATION OF BAL FINDINGS AND OTHER DATA}

In early series, the diagnostic value of BAL findings was considered in isolation in groups of patients, without reference to a priori probabilities of individual diagnoses (based on the relative prevalence of individual disorders), the clinical presentation or findings of other tests. For this reason, these studies fail to quantify the true added diagnostic value of BAL in diffuse parenchymal lung disease. Specifically, the quantification of the added value of a test requires knowledge of the pre-test probability, changes in diagnostic perception resulting from the performance of the test and validation of the accuracy of changes in diagnostic perception against a reference standard.

This methodology, which has been applied to the diagnostic accuracy of HRCT [19], has not been definitively applied to BAL. However, although the accuracy of the first choice diagnosis was not evaluated, a Bayesian analysis established that BAL findings did change average diagnostic likelihoods in a landmark study that included 583 patients with suspected diffuse parenchymal lung disease, examined against final clinical and/or histological diagnoses [20]. Certain BAL profiles resulted in significant changes in diagnostic likelihoods. For example, BAL lymphocyte counts of $>30 \%$ in association with a low granulocyte count increased the probability of sarcoidosis from $34 \%$ (the prevalence of sarcoidosis within the cohort) to $>65 \%$, while reducing the likelihood of IPF from $16 \%$ to $2 \%$. A lymphocyte count of $<30 \%$ in association with an elevated granulocyte count increased the probability of IPF from $16 \%$ to $33 \%$ and radically reduced the likelihood of HP from $9 \%$ to $1 \%$.

These data underline the fact that although BAL might be useful in individual patients, in altering the balance of diagnostic probability, it is not, in isolation, diagnostically decisive in most cases: in clinical practice, diagnostic likelihoods of $30-70 \%$ are synonymous with major uncertainty. The same reservation applied to the predictive value of CD4/ CD8 ratios [20]. When examined in patient subgroups, according to the level of lymphocytosis, high CD4/CD8 ratios conferred diagnostic likelihoods of sarcoidosis of $50-75 \%$, rising to $85 \%$ when associated with a BAL lymphocytosis and low BAL granulocyte count. Similarly, low CD4/CD8 ratios increased the likelihood of HP but only to levels of $20-50 \%$ (depending upon BAL lymphocyte and granulocyte content). As discussed earlier, CD4/CD8 ratios may be useful when applied by experienced diagnosticians but must be integrated with other BAL findings and clinical data, and add little to diagnosis when used in isolation.

The prevailing problem is that no study exists in which BAL data are integrated with the pre-test probability of disease, based not only on disease prevalence, but also on age, sex, smoking history, mode of presentation, observed disease behaviour, observed previous responsiveness to treatment, clinical evaluation and the results of other tests. A computerised 
algorithm has been constructed in which limited clinical information (demographic data, smoking histories and disease prevalence) is integrated with BAL findings, with encouraging rises in diagnostic likelihoods [8]. However, the whole range of information available to the clinician at the time of BAL has not been captured in any study. The problem has been compounded by the advent of HRCT, which has transformed the diagnostic landscape. The definition of pre-BAL diagnostic probabilities has been refined radically in the HRCT era and, thus, the landmark BAL series of the 1980s are now out of date. For example, in groups of patients with a variety of diffuse lung diseases, it is not known whether the presence of a BAL lymphocytosis has diagnostic value which is independent of the presence of HRCT appearances that are diagnostic or suggestive of sarcoidosis or HP. Those who are accustomed to integrating BAL and HRCT findings tend to argue that the two procedures complement each other in diagnostic formulation, but this view needs to be validated. As a result, the added value of BAL, which has made the procedure an important part of the diagnostic algorithm of many experienced practitioners, cannot be readily communicated to less experienced physicians who seek to apply the technique.

A second problem, common to all clinical diagnostic studies, is the issue of an appropriate reference standard. It is sometimes argued that a reference standard is always required in a diagnostic study but this view fails to take into account the realities of clinical practice. If BAL is an integral part of the clinical diagnostic algorithm, when no independent histological diagnosis is available, it becomes impossible to evaluate the diagnostic accuracy of BAL, as BAL findings do, themselves, influence the diagnosis [21]. For all its merits, the study of WELKER et al. [20] does not address the problem of "diagnostic circularity". To overcome this constraint, it is necessary to examine a diagnostic test, not against a reference standard, but by determining whether changes in diagnostic perception conferred by the test are "validated" by corresponding alterations in outcome. This approach was taken recently in a study of the diagnostic significance of a BAL lymphocytosis when the clinical and HRCT presentation is typical of IPF [22]. A BAL lymphocytosis of $>30 \%$ was present in six out of 74 patients and in all six cases an alternative diagnosis to IPF was made. The change in diagnostic perception was validated by surgical biopsy in two cases and by the subsequent outcome (which was not typical of IPF) in four cases. This methodology can only be used when there are clear outcome distinctions between conflicting diagnoses, and is less applicable to the diagnostic distinction between sarcoidosis and $\mathrm{HP}$, for example. However, studies of this sort are needed in order to integrate the use of BAL into modern clinical practice.

\section{BAL IN PROGNOSTIC EVALUATION}

The difficulties in relating historical BAL data to diagnosis apply equally to the use of BAL in the definition of prognosis. In patients with "IPF", a BAL lymphocytosis was found to denote a more favourable course, including a higher likelihood of a response to treatment. By contrast, a BAL neutrophilia or eosinophilia was associated with a poor outcome. However, both observations are difficult to integrate into current practice. The historical entity of "IPF" included entities such as NSIP and COP, characterised by a BAL lymphocytosis and a good outcome. Moreover, it is now recognised that, in a significant minority of patients with chronic HP, there is a histological pattern of usual interstitial pneumonia often without classical HP histological features [23], and it is likely that a number of such cases were included in older "IPF" series. It is increasingly accepted that in diffuse lung disease, "diagnosis is prognosis". Thus, it is unclear whether the prognostic value of a BAL lymphocytosis relates solely to its diagnostic value, or whether it carries a separate prognostic advantage in patients with IPF, diagnosed using recent criteria.

There are similar difficulties in interpreting the prognostic significance of a BAL neutrophilia. It has been argued that a prominent neutrophilia is indicative of a more progressive course in IPF $[24,25]$ and the pulmonary fibrosis of systemic sclerosis (SSc) $[26,27]$. However, in most series, the baseline severity of disease was not taken into account. This is an important caveat. IPF has a much worse outcome than the pulmonary fibrosis of SSc and also has a higher BAL neutrophil content [28]. However, after adjustment for underlying disease severity (using pulmonary function tests and HRCT data in separate multivariate models), BAL neutrophil content does not differ between the two diseases [28], which makes it difficult to argue for a tight link between a BAL neutrophilia and the intrinsic progressiveness of disease. Against this, a BAL neutrophilia was indicative of higher early mortality in a recent IPF study, both before and after adjustment for disease severity [29]. It should be stressed that this finding, in a large cohort, was only weakly statistically significant, underlining the difficulties in applying the findings to routine prognostic evaluation. Furthermore, data are conflicting in IPF diagnosed using modern criteria, with a BAL neutrophilia not linked to mortality in another series [12].

In the pulmonary fibrosis of SSc, a neutrophilia is the most prevalent BAL abnormality and is associated with a worse outcome if other data are not considered. However, there is ample evidence that in SSc, a neutrophilia is linked to more extensive pulmonary fibrosis on HRCT and greater pulmonary function impairment $[30,31]$. In one recent study, the presence of a BAL neutrophilia had no independent prognostic significance in a large cohort of SSc patients, after adjustment for disease severity [30]. In the placebo-controlled oral cyclophosphamide study of STRANGE et al. [32], baseline BAL data were not linked to outcome in the placebo group [32]. Thus, it appears that in SSc, a BAL neutrophilia is no more than a marker of disease severity and the same may also apply to other diffuse lung diseases.

In general, it appears that the presence of a BAL lymphocytosis or neutrophilia is much less influential in prognostic evaluation than accurate diagnosis and the accurate staging of disease severity respectively. However, in highlighting the flaws of historical series containing the more frequent disorders (IPF, sarcoidosis and HP), it is easy to overlook the fact that BAL has a central place in clinical evaluation in a number of specific contexts.

\section{SCENARIOS IN WHICH BAL MAY PLAY A CENTRAL DIAGNOSTIC ROLE}

1) In the correct clinical setting, typical BAL findings are diagnostic in several rare diffuse lung diseases, including 
alveolar proteinosis [33], lipoid pneumonia, acute eosinophilic pneumonia, pulmonary lymphoma [34] and lymphangitis carcinomatosis (although in the last disorder, the diagnosis is usually also apparent at endobronchial or transbronchial biopsy). BAL was also used historically to diagnose Langerhans cell histiocytosis, based on electron microscopic appearances, but this use of BAL is now seldom required as HRCT appearances are usually definitive.

2) BAL is pivotal in excluding or confirming diffuse alveolar haemorrhage in patients with unexplained pulmonary infiltrates. In extensive diffuse alveolar haemorrhage, haemoptysis is often minimal or absent and HRCT findings are highly nonspecific.

3) The diagnosis of opportunistic infection often requires the performance of BAL [35]. When there is major deterioration in patients with diffuse lung disease treated with immunosuppressive therapy, the key management distinction lies between both increasing the level of immunosuppression and treating infection while reducing the level of immunosuppression. This key distinction requires the exclusion of infection on BAL and is often the single most important determinant of the management strategy in this difficult clinical scenario.

4) BAL is occasionally diagnostic when combined with HRCT findings, and this applies to RBILD. The HRCT appearances of RBILD are often indistinguishable from those of HP and, in former smokers, both diagnoses are possible. A BAL finding of an excess of pigmented macrophages without other abnormalities is usually sufficient to clinch the diagnosis of RBILD, without the need for surgical biopsy.

5) Whether or not the presence of a BAL lymphocytosis adds to HRCT data in identifying granulomatous disease, the absence of a BAL lymphocytosis is often useful in excluding atypical HP or sarcoidosis, when these possibilities are raised by HRCT appearances.

6) In suspected drug-induced lung disease, the finding of a prominent BAL eosinophilia usefully increases the diagnostic likelihood, as does the presence of foamy alveolar macrophages in some contexts (e.g. suspected amiodarone-induced lung disease).

\section{SCENARIOS IN WHICH THE VALUE ADDED BY BAL NEEDS FURTHER APPRAISAL}

1) As discussed above, the true clinical utility of BAL in the diagnosis of HP and sarcoidosis needs to be examined with the integration of HRCT data. A computerised diagnostic algorithm, which incorporates demographic and smoking data, does not include HRCT findings [8].

2) The value of BAL in excluding other disorders in the ATS/ERS algorithm for the diagnosis of IPF needs to be evaluated. The recent finding that BAL data may provide accurate change in diagnostic perception when clinical and HRCT features are compatible with IPF [23] needs to be explored further in larger cohorts.

3) The utility of BAL data in delineating the clinico-radiological profile of NSIP for therapeutic purposes needs to be established. The important NSIP dichotomy between patients with a prominent organising pneumonia component and patients with a degree of clinical and HRCT overlap with IPF
[9] requires further evaluation, with exploration of the prognostic value of BAL.

\section{CONCLUSION}

In conclusion, BAL plays a pivotal diagnostic role in a number of specific scenarios, including the diagnosis of several rare disorders and the exclusion of opportunistic infection. It also has a useful ancillary diagnostic role in the more prevalent diffuse parenchymal lung disorders and is particularly useful in reducing the likelihood of HP. However, this role of BAL is not well captured by published studies, most of which predate the reclassification of the IIP. There is now a need for diagnostic and prognostic studies to quantify the value added by BAL to full clinical and HRCT evaluation.

\section{STATEMENT OF INTEREST}

None declared.

\section{REFERENCES}

1 Klech H, Hutter C. Clinical guidelines and indications for bronchoalveolar lavage (BAL). Report of the European Society of Pneumonology Task Group on BAL. Eur Respir J 1990; 3: 937-974.

2 The BAL Cooperative Group Steering Committee: bronchoalveolar lavage constituents in healthy individuals, idiopathic pulmonary fibrosis, and selected comparison groups. Am Rev Respir Dis 1990; 141: S169-S202.

3 Joint American Thoracic Society and European Respiratory Society Group. Idiopathic pulmonary fibrosis: diagnosis and treatment. International consensus statement. Am J Respir Crit Care Med 2000; 161: 646-664.

4 Joint American Thoracic Society and European Respiratory Society Group. International multidisciplinary consensus classification of the idiopathic interstitial pneumonias. Am J Respir Crit Care Med 2002; 165: 277-304

5 Davison AG, Heard BE, McAllister WAC, et al. Cryptogenic organizing pneumonia. Q J Med 1983; 52: 382-394.

6 Ward K, O'Connor C, Odium C, et al. Prognostic value of bronchoalveolar lavage in sarcoidosis: the critical influence of disease progression. Thorax 1989; 44: 6-12.

7 Drent M, Jacobs JA, de Vries J, et al. Does the cellular bronchoalveolar lavage fluid profile reflect the severity of sarcoidosis? Eur Respir J 1999; 13: 1338-1344.

8 Drent M, van Nierop MA, Gerritsen FA, et al. A computer program using BALF-analysis results as a diagnostic tool in interstitial lung diseases. Am J Respir Crit Care Med 1996; 153: 736-741.

9 Travis WD, Hunninghake G, King TE Jr, et al. Idiopathic nonspecific interstitial pneumonia: report of an American Thoracic Society project. Am J Respir Crit Care Med. 2008; 177: 1338-1347.

10 Nagai S, Kitaicha $\mathrm{M}$, Itoh $\mathrm{H}$, et al. Idiopathic nonspecific interstitial pneumonia/fibrosis: comparison with idiopathic pulmonary fibrosis and BOOP. Eur Respir J 1998; 12: 1010-1019.

11 Park JS, Lee KS, Kim JS, et al. NSIP with fibrosis: radiographic and CT findings in seven patients. Radiology 1995; 195: 645-648.

12 Veeraraghavan S, Latsi PI, Wells AU, et al. Bronchoalveolar lavage findings in idiopathic UIP and NSIP. Eur Respir J 2003; 22: 239-244.

13 Wells AU. Cryptogenic organizing pneumonia. Semin Respir Crit Care Med 2001; 22: 449-450.

14 Drent M, van Velzen-Blad H, Diamant M, et al. Relationship between presentation of sarcoidosis and T lymphocyte profile: a study in bronchoalveolar lavage fluid. Chest 1993; 104: 795-800.

15 Drent $M$, van Velzen-Blad $H$, Diamant $M$, et al. Differential diagnostic value of plasma cells in bronchoalveolar lavage fluid. Chest 1993; 103: 1720-1734. 
16 Drent M, Grutters JC, Mulder PG, et al. Is the different $\mathrm{T}$ helper cell activity in sarcoidosis and extrinsic allergic alveolitis also reflected by the cellular bronchoalveolar lavage fluid profile? Sarcoidosis Vasc Diffuse Lung Dis 1997; 14: 31-38.

17 Costabel U, Guzman J. Bronchoalveolar lavage in interstitial lung disease. Curr Opin Pulm Med 2001; 7: 255-261.

18 Kantrow SP, Meyer KC, Kidd P, et al. The CD4/CD8 ratio in BAL fluid is highly variable in sarcoidosis. Eur Respir J 1997; 10: 2716-2721.

19 Aziz ZA, Wells AU, Bateman ED, et al. Clinical decision-making in interstitial lung disease: effect of thin-section CT. Radiology 2006; 238: 725-733.

20 Welker L, Jones RA, Costabel U, et al. Predictive value of BAL cell differentials in the diagnosis of interstitial lung diseases. Eur Respir J 2003; 24: 1000-1006.

21 Kinder BW, Wells AU. The art and science of diagnosing interstitial lung diseases. Am J respir Crit Care Med 2009; 179: 974-975.

22 Oshimo S, Bonella F, Cui A, et al. Significance of bronchoalveolar lavage for the diagnosis of idiopathic pulmonary fibrosis. Am Rev Respir Dis 2009; 179: 1043-1047.

23 Ohtani Y, Saiki S, Kitaichi M, et al. Chronic bird fancier's lung: histopathological and clinical correlation. An application of the 2002 ATS/ERS consensus classification of the idiopathic interstitial pneumonias. Thorax 2005; 60: 665-671.

24 Watters LC, Schwarz MI, Cherniack RM, et al. Idiopathic pulmonary fibrosis: pretreatment bronchoalveolar lavage cellular constituents and their relationships with lung histopathology and clinical response to therapy. Am Rev Respir Dis 1987; 135: 696-704.

25 Boomars KA, Wagenaar SS, Mulder PGH, et al. Relationship between cells obtained by bronchoalveolar lavage and survival in idiopathic pulmonary fibrosis. Thorax 1995; 50: 1087-1092.

26 White B, Moore WC, Wigley FM, et al. Cyclophosphamide is associated with pulmonary function and survival benefit in patients with scleroderma and alveolitis. Ann Intern Med 2000 132: 947-954.

27 Silver RM, Miller KS, Kinsella MB, et al. Evaluation and management of scleroderma lung disease using bronchoalveolar lavage. Am J Med 1990; 88: 470-476.

28 Wells AU, Hansell DM, Haslam PL, et al. Bronchoalveolar lavage cellularity: lone cryptogenic fibrosing alveolitis compared with the fibrosing alveolitis of systemic sclerosis. Am J Respir Crit Care Med 1998; 157: 1474-1482.

29 Kinder BW, Brown KK, Schwarz MI, et al. Baseline BAL neutrophilia predicts early mortality in idiopathic pulmonary fibrosis. Chest 2008; 133: 226-232.

30 Wells AU, Hansell DM, Rubens MB, et al. Fibrosing alveolitis in systemic sclerosis: bronchoalveolar lavage findings in relation to computed tomographic appearances. Am J Respir Crit Care Med 1994; 150: 462-468.

31 Goh NS, Veeraraghavan S, Desai SR, et al. Bronchoalveolar lavage cellular profiles in patients with systemic sclerosis-associated interstitial lung disease are not predictive of disease progression. Arthritis Rheum 2007; 56: 2005-2012.

32 Strange C, Bolster MB, Roth MD, et al. Scleroderma Lung Study Research Group. Bronchoalveolar lavage and response to cyclophosphamide in scleroderma interstitial lung disease. Am J Respir Crit Care Med 2008; 177: 91-98.

33 Martin RJ, Coalson JJ, Rogers RM, et al. Pulmonary alveolar proteinosis: the diagnosis by segmental lavage. Am Rev Respir Dis 1980; 121: 819-825.

34 Betsuyaku T, Munakata M, Yamaguchi S, et al. Establishing diagnosis of pulmonary malignant lymphoma by gene rearrangement analysis of lymphocytes in bronchoalveolar lavage fluid. Am Rev Respir Dis 1994; 149: 526-529.

35 Baughman RP. Use of bronchoscopy in the diagnosis of infection in the immunocompromised host. Thorax 1994; 49: 3-7. 